\title{
Application of proteomics for the identification of biomarkers in amniotic fluid: are we ready to provide a reliable prediction?
}

\author{
George Th. Tsangaris • \\ Athanasios K. Anagnostopoulos • Georgia Tounta • \\ Aris Antsaklis • Ariadni Mavrou • Aggeliki Kolialexi
}

Received: 30 March 2011 / Accepted: 2 May 2011 / Published online: 19 June 2011

(C) European Association for Predictive, Preventive and Personalised Medicine 2011

\begin{abstract}
Proteomics-based identification of biomarkers for fetal abnormalities and pregnancy complications in amniotic fluid (AF) has made significant progress in the past 5 years. This is attributed mainly to advances in mass spectrometry-based proteomic technologies that enable new strategies for discovering biomarkers from complex biological fluids in a high-throughput and sensitive manner. These markers, although they still need to be verified, are diagnostic and may in the future provide targets for therapeutic intervention. In the current review we focus on the emergence of proteomics as a major platform technology in studying AF and developing biomarkers for fetal aneuploidies and pregnancy-related disorders.
\end{abstract}

Keywords Proteomics · Biomarkers · Amniotic fluid · Fetal aneuploidies $\cdot$ Pregnancy complications

G. T. Tsangaris is a National Representative of EPMA in Greece.

G. T. Tsangaris $(\bowtie) \cdot$ A. K. Anagnostopoulos

Proteomics Research Unit, Center of Basic Research II,

Biomedical Research Foundation of the Academy of Athens,

11527 Athens, Greece

e-mail: gthtsangaris@bioacademy.gr

G. Tounta $\cdot$ A. Mavrou $\cdot$ A. Kolialexi

Department of Medical Genetics,

Athens University School of Medicine,

Athens, Greece

\section{A. Antsaklis}

1st Department of Obstetrics \& Gynecology,

Athens University School of Medicine,

Athens, Greece

\section{Introduction}

Over the last decade, research mainly focused on the molecular characterization of various biological systems and disease states. The human genome consists of approximately 21.000 genes and provides researchers with valuable gene expression data. It is the human proteome, however, that actually regulates cellular function and ultimately determines the phenotype. The proteome is dynamic and changes constantly under the control of a particular set of intrinsic and extrinsic stimuli. This process is complicated because of the ability of the gene to produce more than one protein, posttranslational processing and structural modifications which lead to functional proteins [1].

Proteomic analysis has become a popular platform for the discovery of biomarkers for various pathologies using several types of biological fluids or tissues [2-4]. Proteomic approaches are already used in some fields of medical research, including liver, heart disease, certain forms of cancer and pregnancy research [5-7].

Pregnancy and proteomics

Pregnancy progression and delivery rely on complex fetomaternal processes that involve interactions of intracellular and extracellular factors including hormones, adhesion molecules, growth factors and immunomodulators [8]. Maternal physiological processes and immune responses are modified to allow the semi-allogenic fetus to survive within the uterine environment [9]. Fetal tissues, on the other hand, serve as endocrine organs and produce a wide range of proteins that enter maternal and fetal circulation 
and have diverse effects on various systems [10]. An intricate balance of these substances is required throughout pregnancy and in cases of gestational disease or fetal genetic abnormalities this balance may be disturbed. Identification of proteins specific to pregnancy is likely to contribute to the comprehension of the underlying pathophysiology and to the discovery of relevant biomarkers for fetal aneuploidies and pregnancy complications $[3,4,11]$. If detected in early pregnancy or before the development of clinical symptoms they can be used as suitable disease markers, whereas those detected at later stages are likely to be more specific and may be closely related to the phenotype of the disease. Biomarkers can also improve management of pregnancy, determine the outcome and allow plan for possible complications in delivery.

This article aims to summarize applications of proteomic technology on amniotic fluid (AF), the most important and specific body fluid related to gestation. With the advent of high resolution ultrasonography, AF is accessible for extensive studies and application of proteomic methods has yielded significant information regarding the physiology of reproduction and pathological conditions related to pregnancy. A considerable amount of data are now available on several aspects including pregnancy progression and delivery.

\section{Physiology of amniotic fluid}

Amniotic fluid (AF) is fundamental for the normal development of the human fetus during pregnancy. The amniotic sac, which contains the embryo, forms about 12 days after conception. AF immediately begins to fill the sac and at this time it consists mainly of water supplied by the mother [12, 13]. After approximately 12 weeks, fetal urine makes up most of the fluid. At 15 weeks of gestation, the volume of $\mathrm{AF}$ is about $200 \mathrm{ml}$, at 24 weeks about $800 \mathrm{ml}$ and continues to increase until the 28 to 32 week of pregnancy. After that time, the level of AF generally stays the same until the 37-40 weeks when the level declines [14].

$\mathrm{AF}$ is a rich source of biomarkers for the diagnosis of fetal genetic abnormalities and pregnancy complications. The biochemical composition of the AF is complex, varies throughout pregnancy and is mainly related to functions of the different fetal and amniotic compartments. It contains proteins, amino acids, carbohydrates, hormones, lipids and electrolytes. In contrast to other biological fluids, like plasma and serum, AF has a low protein concentration and relatively high carbohydrate and lipid content. Many of the protein molecules present in AF are structurally glycoconjugates and are protected against proteolytic attack from the extracellular matrix proteases [15-17]. Many proteins detected in the $\mathrm{AF}$ are already present at a very early stage of gestation, whereas others are detected only at the end of pregnancy. The concentration of a given protein in the $\mathrm{AF}$ is governed not only by fetal, placental, or maternal synthesis and degradation, but also by exchanges between the mother and the fetus through the placenta. Fetomaternal transfer of proteins involves several different mechanisms. Consequently, the concentration of each AF protein results from a balance between opposing dynamic metabolic and physiological processes which proceed simultaneously.

Because of these particular characteristics several variables need to be considered for $\mathrm{AF}$ effective proteomic analysis, including sample preparation and handling, protein pre-fractionation, affinity depletion of highly abundant proteins, isolation of subproteomes (e.g. glycoproteome and phosphoproteome), multidimensional chromatographic separation etc. Prior to proteome analysis it is necessary to measure protein concentration of the sample. Two methods seem to be effective and are widely used: a) precipitation of the protein content using an organic solvent, mainly acetonitrile and b) concentration of the sample with ultracentrifugal filters with a molecular weight cut off $<10 \mathrm{kDa}[18-20]$.

Mass spectrometry-based proteomics (MS) has become the method of choice for the analysis of AF. The advantages of MS include sensitivity, resolution, speed and high throughput, but at the expense of generating huge amount of data to be analyzed using more advanced bioinformatics. The choice of MS-based strategy is strongly dependent on the biological question to be addressed, and also on the specificity and the amount of material available. Proteomic techniques used for the analysis of AF could be divided in two major groups, namely «top-down» and «bottom-up» $[21,22]$. In the former, intact proteins are characterized directly via MS, whereas in the latter, proteins are first digested and the resulting peptides are then analyzed to identify and sometimes quantify proteins (the «bottom-up» approach is frequently used with liquid chromatography).

Techniques employing gels and gel independent methods have been applied for the analysis of AF. The most common and widely accepted approach relies upon a coordinated use of 2-DE, image analysis, mass spectrometric protein identification, and bioinformatics/database construction [23, 24]. Gelfree electrophoresis, which allows sample fractionation by isoelectric focusing, trypsin digestion of the fragments and finally peptide analysis by capillary LC-MS/MS was also introduced for the analysis of AF $[18,25]$.

\section{The normal human amniotic fluid proteome}

Published proteome analyses of human AF were originally limited to cultured cells. Specifically, Nilsson et al. by 
direct LC analysis of digested samples, followed by Fourier transform ion cyclotron resonance MS, identified 43 proteins in the 15th-week AF [26]. Oh et al. investigated metabolic enzymes in cultured AF cells and reported on a possible screening method for metabolic enzymes and metabolism related proteins which might form the basis for future metabolic screening when amniocentesis is carried out [27]. Jin et al. investigated the influence of the exposure of AF cells to N-methyl-N'-nitro-N-nitrosoguanidine, known to induce DNA damage, leading to chromosome rearrangements and reported significant changes in the proteome profile [28].

Since 1997 the proteomic profile of the normal human AF was determined by several groups. Liberatori et al. identified human AF proteins in uncultured AF supernatant by immunoblot analysis and reported a $2 \mathrm{D}-\mathrm{E}$ protein map of human AF obtained at the 17th-week of gestation, when $\mathrm{AF}$ is most commonly obtained for prenatal diagnosis of chromosomal abnormalities by amniocentesis [24]. Tsangaris et al. using 2D-E coupled with MALDI-TOF-MS, published a protein database of normal human AF cells consisting of 432 different gene products [29]. In another study, Tsangaris et al., reported on the proteomic profile of the AF supernatant comprising of 136 different gene products (Fig. 1) [30]. Queloz et al. compared the proteomic profiles of normal AF obtained at 17 and 40 weeks of pregnancy using 2-DE and silver staining, as well as $2 \mathrm{D}-$ DIGE [31]. Results showed that some proteins were more abundant early in pregnancy, while others were overexpressed at term, suggesting that the protein profile of $\mathrm{AF}$ is dynamic and changes occur during development. These observations are particularly important when biomarkers for a specific condition are identified. In 2007, Cho et al. reported on the most extensive protein profile of the second trimester normal human AF, which is comprised of 1026 unique gene products from 842 different genes [32]. It is interesting to note that the protein list reported in this study includes hCG- $\beta$ chain, AFP and inhibin $A$, which are currently used as serum screening markers for Down syndrome (DS).

\section{Amniotic fluid proteomic studies for the detection of fetal aneuploidies}

In an attempt to identify novel biomarkers for fetal chromosomal abnormalities several proteomic studies have been performed using AF samples obtained from cases known to carry chromosomally abnormal fetuses. Although in preliminary proteomic studies, no relevant protein markers had been
Fig. 1 Two dimensional gel analysis of normal human AF supernatant. (From [30], copyright IIAR. Reproduced with permission)

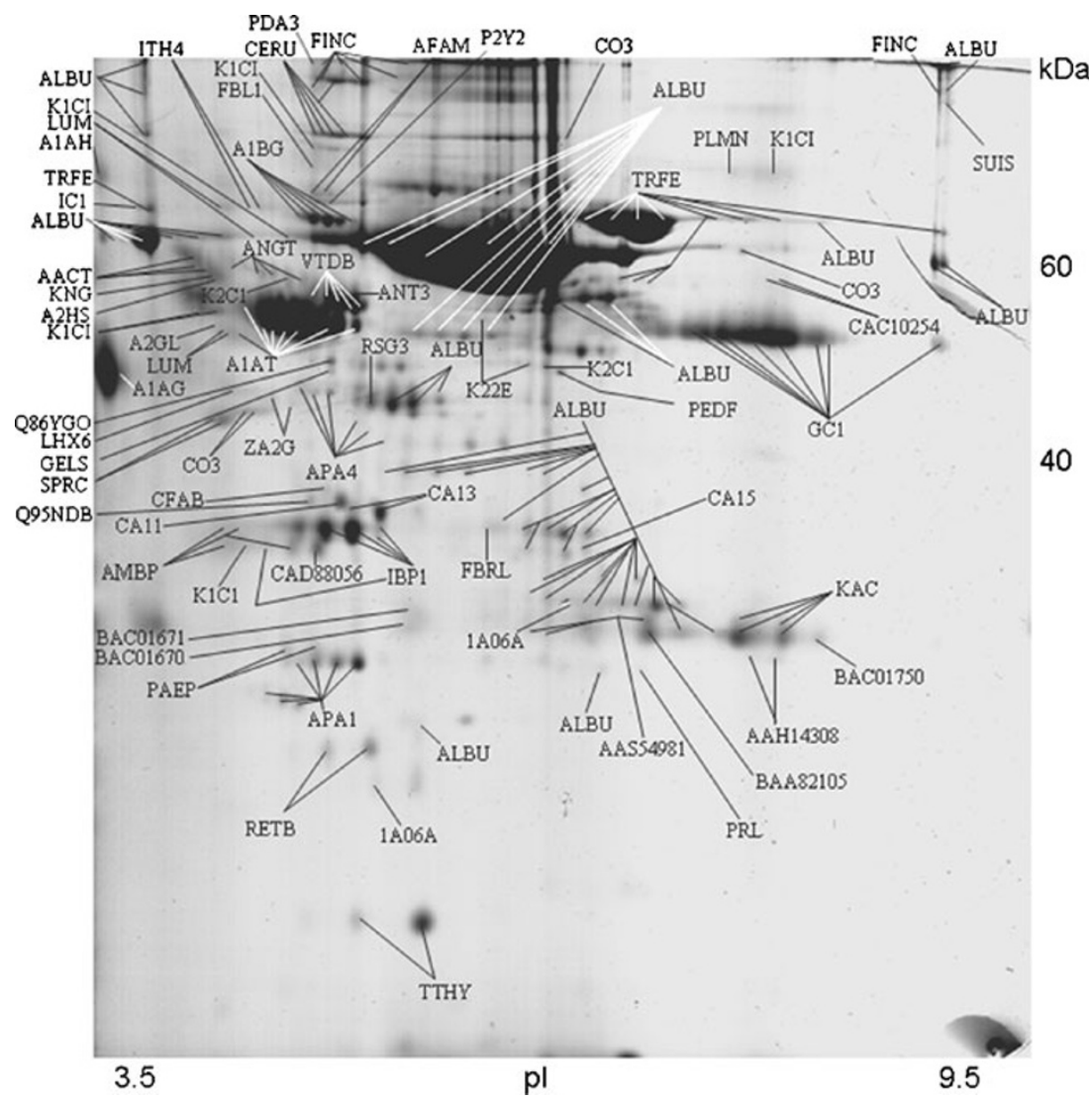


validated for prenatal diagnosis of fetal pathological conditions, recent publications have demonstrated that proteomic tools could be of potential clinical value.

An AF fingerprint was generated by Wang et al. using a large variety of surface arrays in 20 samples obtained from pregnant women known to carry an aneuploid fetus [33]. Following application of pattern recognition algorithms, it was possible to successfully identify aneuploidies, based on distinct biomarker peaks that segregated at $2.65-7.0 \mathrm{kd}$, but it was not possible to differentiate between the various chromosome abnormalities.

Since DS is the most common chromosomal abnormality with a prevalence of 1 in 732 fetuses, the majority of studies focus on the detection of biomarkers for this congenital anomaly. Oh et al. identified metabolic changes in AF samples coming from pregnancies carrying DS fetuses in a study concerning use of proteomic techniques for the identification of metabolic changes [27]. Specifically he noted significant derangement in the carbohydrate and amino acid handling, purine and intermediary metabolism as well as miscellaneous metabolic pathways. Tsangaris et al. identified biomarkers in AF samples from pregnancies with DS fetuses and chromosomally normal ones [34]. This comparison revealed seven proteins differentially expressed in samples obtained from pregnancies with DS fetuses as compared to controls. Alpha1-microglobulin, collagen alpha 1 (I) chain, collagen alpha 1 (III) chain, collagen alpha 1 (V) chain $\mathrm{d}$ and basement membrane-specific heparin sulfate proteoglycan core protein were increased in cases with DS, whereas insulin-like growth factor-binding protein was decreased by $40 \%$ compared to chromosomally normal fetuses. In the same study, splicing factor arginine/ serine-rich protein was present only in AF samples coming from cases with DS syndrome fetuses and was completely absent in the chromosomally normal ones. It is important to note that genes encoding for these proteins are located on chromosomes other than chromosome 21, but their expression is possibly regulated by transcription factors located on chromosome 21.

In another study the same group identified in $\mathrm{AF}$ samples obtained from pregnancies with Turner syndrome fetuses seven biomarkers for Turner syndrome, using the same MS based technology [35]. Serotransferin, lumican, plasma retinol-binding protein and apolipoprotein A-I were increased in Turner syndrome, while kininogen, prothrombin, and apolipoprotein A-IV were decreased. Interestingly, APOA1 and plasma retinol-binding protein were also altered in $\mathrm{AF}$ obtained from cases with Klinefelter syndrome fetuses [36].

Mange et al. described a proteomic approach combining ProteinChip technology that allowed screening for fetal aneuploidies in AF with prediction accuracy of approximately $90 \%$, that might be helpful for the rapid clinical management of high-risk pregnancies for fetal aneuploidies [37].
In 2009 Wang et al. identified proteins differentially expressed in AF coming from pregnancies with DS and trisomy 18 fetuses [38]. The proteins with significant differential expression in samples obtained from cases with DS fetuses were APOA1, SERPINA3, prealbumin (transthyretin, TTR) and transferrin (TF). Levels of apolipoprotein A1, AP-3mu and antitrypsin were significantly decreased in trisomy-18 AF, whereas placental protein-14 was increased. On the other hand, apolipoprotein A1 was decreased in trisomy-21 AF, but antitrypsin, prealbumin and transferrin were increased. Proteins differentially expressed in samples coming from women carrying trisomy 18 fetuses were involved in immune processes, dysfunction of skin pigmentation and platelet disorders, whereas those of DS were associated with dysfunctional lipid and cholesterol metabolism, processes of metal ion transport, adenosine triphosphate metabolism and energy coupled protein transport.

Cho et al. used two-dimensional LC, followed by MS/MS, to analyze AF of chromosomally normal and DS-affected pregnancies [39]. A total of 542 proteins were identified by LTQ-Orbitrap mass spectrometer and data were compared semi-quantitatively by spectral counting. Based on the spectral count differences between the two groups analyzed, after normalization, 60 candidate biomarkers that showed greater than two-fold increase or decrease in concentration in the presence of DS fetuses were identified. The differential expression of amyloid precursor protein and tenascin- $\mathrm{C}$ were verified by ELISA and both showed a two-fold increase, on average, in DS-AF samples as compared to controls. Seven proteins from chromosome 21 were identified in this study and all showed increased expression in DS AF as compared to controls, supporting the long-standing «gene-dosage hypothesis».

Recently, Park et al. analyzed AF samples collected in the 2 nd trimester of pregnancy by liquid chromatographyelectrospray ionization-tandem mass spectrometry (LCESI-MS/MS) [40]. Thirty new AF proteins were identified in this study and $44 \mathrm{AF}$ proteins were found to be differentially expressed in DS and normal cases. Six of these proteins were unique to DS AF, while 11 were unique to chromosomally normal cases. In addition, 19 proteins were down-regulated and 8 were up-regulated in DS cases with varying fold changes. Western blot analysis confirmed the LC-ESI-MS/MS data that detection of Apolipoprotein combination with A-II and AFP could represent a potential tool for diagnosing DS cases.

\section{Proteome studies in pregnancy related complications}

Pregnancy related disorders such as intra uterine growth restriction (IUGR), pre-eclampsia (PE), preterm labor (PTL) and intra amniotic infection (IAI) contribute signif- 
icantly to maternal and fetal mortality. Each of these disorders is considered to have multifactorial etiology, has a prevalence of $5-10 \%$, and is often difficult to predict [ 41 , 42]. Although several pathways for the pathogenesis of pregnancy complications have been proposed, the basic molecular mechanisms that modulate these events remain incompletely understood. Discovery of clinically and biologically relevant biomarkers able to reveal key pathogenic pathways and predict pregnancies at risk for antenatal fetal damage is a priority. Proteomics provides a unique opportunity to fill this gap.

\section{Biomarkers for the early prediction of pre-eclampsia}

$\mathrm{PE}$ is a complex disorder of pregnancy, which is characterized by hypertension and proteinuria that develop after 20 weeks of gestation in previously normotensive women [43]. Up to date, no therapeutic approaches are available for either treatment or prevention of PE. This rapidly progressive syndrome is diagnosed when the mother develops hypertension and proteinuria. The only cure for PE is delivery, resulting in a third of the babies being born prematurely. It is estimated that more than 8 million women are affected each year and over 70.000 maternal deaths occur worldwide. Therefore, biomarkers are important to help understand disease, potentially identify women at risk to improve their outcomes and design therapies.

There are only a few reports in the literature on the use of proteomic analysis in pre-eclampsia. In an effort to elucidate the pathogenesis of PE, Vascotto et al. compared the amniotic fluid proteomic maps of five PE patients with those of five controls by two-dimensional electrophoresis (2D-E), followed by peptide mapping and tandem mass spectrometric analysis [44]. Results showed that transthyretin and retinol-binding protein were present in $\mathrm{AF}$ samples of women who had developed PE and control women as a mixture of dimeric and post-translationally modified monomeric forms. Although the nature of these forms was similar in both groups, women who had developed PE showed a significant increase in the amount of monomeric proteins with respect to controls, possibly due to the higher oxidative stress associated with PE.

In another study, Park et al. using SELDI TOF MS identified proapolipoprotein A-I and a functionally obscure peptide, SBBI42 (peak X) that can be used to distinguish PE from chronic hypertension and normotensive controls [45].

Wang et al. reported leptin as a predictive marker for PE, since significant elevation of this protein was measured in AF samples 2 months earlier than the appearance of symptoms [46]. In another study the same group reported increased levels of $\mathrm{F} 2$-isoprostane in $\mathrm{AF}$ in the women who later developed pre-eclampsia [47]. Since F2-isoprostane is a marker of oxidative stress and lipid peroxidation this finding may be used as a guide for antioxidant supplementation to reduce the risk and/or severity of preeclampsia.

Potential biomarkers for the early diagnosis of preterm labor and intra amniotic infection

PTL and adverse neonatal outcome are strongly associated with the presence of clinically undetectable IAI.

Use of surface-enhanced laser desorption ionization time of flight (SELDI-TOF) analysis coupled with LC-MS/MS for AF profiling revealed differences in the peak intensity in AF samples obtained from women with PTL and subclinical IAI [48]. Based on the results of this study, calgranulin B and a proteolytic fragment IGFBP-1 have been proposed as candidate biomarkers for IAI. It is of special interest that the same differences in protein expression were also found in maternal serum, allowing for non-invasive detection of IAI. The role of IGFBP-1 biological activity in IAI was recently confirmed by Bujold et al. using a combination of techniques involving 2D chromatography, MS and immunoassays [49].

Buhimschi et al. profiled specific proteins for inflammation in $\mathrm{AF}$ and reported on the existence of four biomarkers (defensins-2 and -1 , calgranulin-C, and calgranulin-A), which were called the «MR score», that can quickly and accurately detect potentially dangerous infections in pregnant women and predict premature birth [50-52]. The «MR score» presents a gradient of disease activity and progresses from absent to mild and severe inflammation. If no biomarkers are present, then the pregnancy is considered to be uncomplicated while an «MR score» of three or four is highly predictive of adverse pregnancy outcome. Thus, biomarkers that constitute the «MR score» can identify patients who might benefit from in utero interventions in a modern diagnostic-therapeutic framework.

Romero et al. using liquid chromatography tandem mass spectrometry with isobaric labeling (iTRAQ) of the AF proteome of women with PTL identified proteins differentially regulated in women with IAI and in those without IAI who delivered preterm [22]. Importantly, many novel proteins were found to be up-regulated in the AF of patients with PTL and IAI including leukocyte elastase precursor, Thymosin-like 3, and 14-3-3 protein isoforms. Moreover, they observed differential expression of proteins in $\mathrm{AF}$ of patients who delivered preterm in the absence of IAI as compared to those with PTL who delivered at term including Mimecan precursor, latenttransforming growth factor b-binding protein isoform $1 \mathrm{~L}$ precursor, and Resistin.

Park et al. found altered expression of Calgranulin A and $\mathrm{B}$ in human amnion and $\mathrm{AF}$ samples obtained from pregnant women infected with Ureaplasma urealyticum, but not in any of the patients without infection [53]. 
Ruetschi et al. analyzed AF from pregnant women with clinical signs of PLT with or without IAI/infection using SELDI-TOF and identified 17 proteins significantly overexpressed in pregnancies complicated with inflammation [54]. Peptide mass fingerprinting followed by Western blotting and ELISA confirmed that five of the biomarkers (human neutrophil defensins 1-3, and calgranulin-A and -B) indicate intra-amniotic inflammation and allow the discrimination between PRL and PROM. Furthermore, the authors applied the MR scoring system to evaluate the diagnostic potential of the 4-peak panel previously described.

Vuadens et al. identified new potential markers for PROM [55]. Their study was based on 2-DE differential display between $\mathrm{AF}$ and plasma samples in the 17th week of gestation, followed by micro-sequencing analysis of specific spots. Two peptides corresponding to the $\mathrm{COOH}-$ terminus fragment of agrin and perlecan which were present only in AF but absent from plasma samples of pregnant women have been considered as possible biomarkers for PROM. These results were further confirmed by Thadikkaran et al. [56]. Michel et al. applied off-gel isoelectric focusing technique followed by tryptic digestion of the proteins and by LC-MS/MS to analyze the plasma and AF sample from a woman at pregnancy term [18]. Systematic comparison revealed that nineteen proteins were specifically present in the AF and absent in maternal plasma. Among them ten had been previously described as pregnancy or placenta specific and could therefore be further characterized as potential PROM biomarkers. No significant relationship, however regarding the sensitivity, specificity and predictive value for the accurate detection of women at risk for PROM has been demonstrated up to now.

\section{Concluding remarks and outlook}

A number of putative biomarkers for fetal aneuploidies and pregnancy complications have been discovered from analysis of human AF. These biomarkers can be used to discriminate cases complicated or women carrying chromosomally abnormal fetuses, to provide insight into involvement of pathophysiological pathways and may in the future provide targets for therapeutic intervention.

Although very promising, some of the candidate biomarkers are relatively abundant, shared among different types of pathologies and overexpressed in other human diseases. Future validation of candidate biomarkers on large independent patient cohorts is needed to quantify protein biomarkers in a sufficient number of samples and determine which may discriminate the presence or absence of disease.

\section{References}

1. Winslow RL, Boguski MS. Genome informatics: current status and future prospects. Circ Res. 2003;92:953-61.

2. Kulasingam V, Diamandis EP. Proteomics analysis of conditioned media from three breast cancer cell lines: a mine for biomarkers and therapeutic targets. Mol Cell Proteomics. 2007;6:1997-2011.

3. Brewis IA. Proteomics in reproductive research: the potential importance of proteomics to research in reproduction. Hum Reprod. 1999;14:2927-9.

4. Page NM, Kemp CF, Butlin DJ, Lowry PJ. Placental peptides as markers of gestational disease. Reproduction. 2002;123:487-95.

5. Rockett JC. Genomic and proteomic techniques applied to reproductive biology. Genome Biol. 2001; 2:REPORTS4020.

6. Coonrod SA, Wright PW, Herr JC. Oolemmal proteomics. J Reprod Immunol. 2002;53:55-65.

7. Romero R, Kuivaniemi H, Tromp G. Functional genomics and proteomics in term and preterm parturition. J Clin Endocrinol Metab. 2002;87:2431-4.

8. Pellicer A, Albert C, Mercader A, Bonilla-Musoles F, Remohi J, Simon C. The pathogenesis of ovarian hyperstimulation syndrome: in vivo studies investigating the role of interleukin-1beta, interleukin-6, and vascular endothelial growth factor. Fertil Steril. 1999;71:482-9.

9. Shankar R, Cullinane F, Brennecke SP, Moses EK. Applications of proteomic methodologies to human pregnancy research: a growing gestation approaching delivery? Proteomics. 2004;4:1909-17.

10. Kim YS, Kim MS, Lee SH, Choi BC, Lim JM, Cha KY, et al. Proteomic analysis of recurrent spontaneous abortion: identification of an inadequately expressed set of proteins in human follicular fluid. Proteomics. 2006;6:3445-54.

11. Jauniaux E, Greenwold N, Hempstock J, Burton GJ. Comparison of ultrasonographic and Doppler mapping of the intervillous circulation in normal and abnormal early pregnancies. Fertil Steril. 2003;79:100-6.

12. Brace RA. Physiology of amniotic fluid volume regulation. Clin Obstet Gynecol. 1997;40:280-9.

13. Sohaey R. Amniotic fluid and the umbilical cord: the fetal milieu and lifeline. Semin Ultrasound CT MR. 1998;19:355-69.

14. Sherer DM. A review of amniotic fluid dynamics and the enigma of isolated oligohydramnios. Am J Perinatol. 2002;19:253-66.

15. Irving-Rodgers HF, Roger J, Luck MR, Rodgers RJ. Extracellular matrix of the corpus luteum. Semin Reprod Med. 2006;24:24250.

16. Orczyk-Pawilowicz M, Florianski J, Zalewski J, Katnik-Prastowska I. Relative amounts of sialic acid and fucose of amniotic fluid glycoconjugates in relation to pregnancy age. Glycoconj J. 2005;22:433-42.

17. Yeung WS, Lee KF, Koistinen R, Koistinen H, Seppala M, Ho $\mathrm{PC}$, et al. Roles of glycodelin in modulating sperm function. Mol Cell Endocrinol. 2006;250:149-56.

18. Michel PE, Crettaz D, Morier P, Heller M, Gallot D, Tissot JD, et al. Proteome analysis of human plasma and amniotic fluid by Off-Gel isoelectric focusing followed by nano-LC-MS/MS. Electrophoresis. 2006;27:1169-81.

19. Polson C, Sarkar P, Incledon B, Raguvaran V, Grant R. Optimization of protein precipitation based upon effectiveness of protein removal and ionization effect in liquid chromatographytandem mass spectrometry. J Chromatogr B Analyt Technol Biomed Life Sci. 2003;785:263-75.

20. Merrell K, Southwick K, Graves SW, Esplin MS, Lewis NE, Thulin CD. Analysis of low-abundance, low-molecular-weight serum proteins using mass spectrometry. J Biomol Tech. 2004;15:238-48. 
21. Han X, Aslanian A, Yates 3rd JR. Mass spectrometry for proteomics. Curr Opin Chem Biol. 2008;12:483-90.

22. Romero R, Kusanovic JP, Gotsch F, Erez O, Vaisbuch E, MazakiTovi S, et al. Isobaric labeling and tandem mass spectrometry: a novel approach for profiling and quantifying proteins differentially expressed in amniotic fluid in preterm labor with and without intraamniotic infection/inflammation. J Matern Fetal Neonatal Med. 2010;23:261-80.

23. Fountoulakis M, Langen H. Identification of proteins by matrixassisted laser desorption ionization-mass spectrometry following in-gel digestion in low-salt, nonvolatile buffer and simplified peptide recovery. Anal Biochem. 1997;250:153-6.

24. Liberatori S, Bini L, De Felice C, Magi B, Marzocchi B, Raggiaschi R, et al. A two-dimensional protein map of human amniotic fluid at 17 weeks' gestation. Electrophoresis. 1997;18:2816-22.

25. Heller M, Michel PE, Morier P, Crettaz D, Wenz C, Tissot JD, et al. Two-stage Off-Gel isoelectric focusing: protein followed by peptide fractionation and application to proteome analysis of human plasma. Electrophoresis. 2005;26:1174-88.

26. Nilsson S, Ramstrom M, Palmblad M, Axelsson O, Bergquist J. Explorative study of the protein composition of amniotic fluid by liquid chromatography electrospray ionization Fourier transform ion cyclotron resonance mass spectrometry. J Proteome Res. 2004;3:884-9.

27. Oh JE, Fountoulakis M, Juranville JF, Rosner M, Hengstschlager M, Lubec G. Proteomic determination of metabolic enzymes of the amnion cell: basis for a possible diagnostic tool? Proteomics. 2004;4:1145-58.

28. Jin J, Gao Z, Guo L, Yang J, Yu Y. Altered expression of zinc finger proteins, ADAMs, and integrin-related proteins following treatment of cultured human cells with a low concentration of Nmethyl-N'-nitro-N-nitrosoguanidine. Environ Mol Mutagen. 2003;41:344-52.

29. Tsangaris G, Weitzdorfer R, Pollak D, Lubec G, Fountoulakis M. The amniotic fluid cell proteome. Electrophoresis. 2005;26:1168-73.

30. Tsangaris GT, Kolialexi A, Karamessinis PM, Anagnostopoulos AK, Antsaklis A, Fountoulakis M, et al. The normal human amniotic fluid supernatant proteome. In Vivo. 2006;20:479-90.

31. Queloz PA, Crettaz D, Thadikkaran L, Sapin V, Gallot D, Jani J, et al. Proteomic analyses of amniotic fluid: potential applications in health and diseases. J Chromatogr B Analyt Technol Biomed Life Sci. 2007;850:336-42.

32. Cho CK, Shan SJ, Winsor EJ, Diamandis EP. Proteomics analysis of human amniotic fluid. Mol Cell Proteomics. 2007;6:1406-15.

33. Wang TH, Chang YL, Peng HH, Wang ST, Lu HW, Teng SH, et al. Rapid detection of fetal aneuploidy using proteomics approaches on amniotic fluid supernatant. Prenat Diagn. 2005;25:559-66.

34. Tsangaris GT, Karamessinis P, Kolialexi A, Garbis SD, Antsaklis A, Mavrou A, et al. Proteomic analysis of amniotic fluid in pregnancies with Down syndrome. Proteomics. 2006;6:4410-9.

35. Mavrou A, Anagnostopoulos AK, Kolialexi A, Vougas K, Papantoniou N, Antsaklis A, et al. Proteomic analysis of amniotic fluid in pregnancies with Turner syndrome fetuses. J Proteome Res. 2008;7:1862-6.

36. Anagnostopoulos AK, Kolialexi A, Mavrou A, Vougas K, Papantoniou N, Antsaklis A, et al. Proteomic analysis of amniotic fluid in pregnancies with Klinefelter syndrome foetuses. J Proteomics. 2009;73:943-50.

37. Mange A, Desmetz C, Bellet V, Molinari N, Maudelonde T, Solassol J. Proteomic profile determination of autosomal aneuploidies by mass spectrometry on amniotic fluids. Proteome Sci. 2008;6:1.

38. Wang TH, Chao AS, Chen JK, Chao A, Chang YL, Cheng PJ, et al. Network analyses of differentially expressed proteins in amniotic fluid supernatant associated with abnormal human karyotypes. Fertil Steril. 2009;92:96-107.

39. Cho CK, Smith CR, Diamandis EP. Amniotic fluid proteome analysis from Down syndrome pregnancies for biomarker discovery. J Proteome Res. 9:3574-82

40. Park J, Cha DH, Jung JW, Kim YH, Lee SH, Kim Y, et al. Comparative proteomic analysis of human amniotic fluid supernatants with down syndrome using mass spectrometry. J Microbiol Biotechnol. 2010;20:959-67.

41. Lumley J. Defining the problem: the epidemiology of preterm birth. BJOG. 2003;110 Suppl 20:3-7.

42. The hypertensive disorders of pregnancy. Report of a WHO study group. World Health Organ Tech Rep Ser. 1987;758:1-114.

43. Dekker GA. Risk factors for preeclampsia. Clin Obstet Gynecol. 1999;42:422-35.

44. Vascotto C, Salzano AM, D'Ambrosio C, Fruscalzo A, Marchesoni $\mathrm{D}$, di Loreto $\mathrm{C}$, et al. Oxidized transthyretin in amniotic fluid as an early marker of preeclampsia. J Proteome Res. 2007;6:160-70.

45. Park JS, Oh KJ, Norwitz ER, Han JS, Choi HJ, Seong HS, et al. Identification of proteomic biomarkers of preeclampsia in amniotic fluid using SELDI-TOF mass spectrometry. Reprod Sci. 2008;15:457-68.

46. Wang CN, Chang SD, Peng HH, Lee YS, Chang YL, Cheng PJ, et al. Change in amniotic fluid levels of multiple anti-angiogenic proteins before development of preeclampsia and intrauterine growth restriction. J Clin Endocrinol Metab. 2010;95:1431-41.

47. Wang CN, Chen JY, Sabu S, Chang YL, Chang SD, Kao CC, et al. Elevated amniotic fluid $\mathrm{F}(2)$-isoprostane: A potential predictive marker for preeclampsia. Free Radic Biol Med. 2011;50:1124-30.

48. Gravett MG, Novy MJ, Rosenfeld RG, Reddy AP, Jacob T, Turner $\mathrm{M}$, et al. Diagnosis of intra-amniotic infection by proteomic profiling and identification of novel biomarkers. JAMA. 2004;292:462-9.

49. Bujold E, Romero R, Kusanovic JP, Erez O, Gotsch F, Chaiworapongsa $\mathrm{T}$, et al. Proteomic profiling of amniotic fluid in preterm labor using two-dimensional liquid separation and mass spectrometry. J Matern Fetal Neonatal Med. 2008;21:697-713.

50. Buhimschi CS, Buhimschi IA, Abdel-Razeq S, Rosenberg VA, Thung SF, Zhao G, et al. Proteomic biomarkers of intra-amniotic inflammation: relationship with funisitis and early-onset sepsis in the premature neonate. Pediatr Res. 2007;61:318-24.

51. Buhimschi CS, Dulay AT, Abdel-Razeq S, Zhao G, Lee S, Hodgson EJ, et al. Fetal inflammatory response in women with proteomic biomarkers characteristic of intra-amniotic inflammation and preterm birth. BJOG. 2009;116:257-67.

52. Buhimschi CS, Rosenberg VA, Dulay AT, Thung S, Sfakianaki AK, Bahtiyar MO, et al. Multidimensional system biology: genetic markers and proteomic biomarkers of adverse pregnancy outcome in preterm birth. Am J Perinatol. 2008;25:175-87.

53. Park SJ, Yoon WG, Song JS, Jung HS, Kim CJ, Oh SY, et al. Proteome analysis of human amnion and amniotic fluid by twodimensional electrophoresis and matrix-assisted laser desorption/ ionization time-of-flight mass spectrometry. Proteomics. 2006;6:349-63.

54. Ruetschi U, Rosen A, Karlsson G, Zetterberg H, Rymo L, Hagberg H, et al. Proteomic analysis using protein chips to detect biomarkers in cervical and amniotic fluid in women with intraamniotic inflammation. J Proteome Res. 2005;4:2236-42.

55. Vuadens F, Benay C, Crettaz D, Gallot D, Sapin V, Schneider P, et al. Identification of biologic markers of the premature rupture of fetal membranes: proteomic approach. Proteomics. 2003;3:1521-5.

56. Thadikkaran L, Crettaz D, Siegenthaler MA, Gallot D, Sapin V, Iozzo RV, et al. The role of proteomics in the assessment of premature rupture of fetal membranes. Clin Chim Acta. 2005;360:2736. 